
\title{
25 Research Soure \\ T cell immunoglobulin and mucin domain- 3 is associated with disease activity and progressive joint damage in rheumatoid arthritis patients
}

Haruki Matsumoto

Fukushima Kenritsu Ika Daigaku

Yuya Fujita

Fukushima Kenritsu lka Daigaku

Tomoyuki Asano

Fukushima Kenritsu Ika Daigaku

Naoki Matsuoka

Fukushima Kenritsu Ika Daigaku

Jumpei Temmoku

Fukushima Kenritsu Ika Daigaku

Shuzo Sato

Fukushima Kenritsu Ika Daigaku

Makiko Yashiro Furuya

Fukushima Kenritsu lka Daigaku

Hiroshi Watanabe

Fukushima Kenritsu Ika Daigaku

kiyoshi migita ( $\nabla$ migita@fmu.ac.jp )

fukuhima medical university

\section{Research article}

Keywords: anti-citrullinated peptide antibody, matrix metalloproteinase-3, rheumatoid arthritis, $\mathrm{T}$ cell immunoglobulin and mucin domain-3

Posted Date: June 8th, 2020

DOI: https://doi.org/10.21203/rs.3.rs-31887/v1

License: (1) (1) This work is licensed under a Creative Commons Attribution 4.0 International License.

Read Full License 
Version of Record: A version of this preprint was published at Medicine on October 30th, 2020. See the published version at https://doi.org/10.1097/MD.0000000000022892. 


\section{Abstract \\ Background}

T cell immunoglobulin and mucin domain-3 (TIM-3) is a surface molecules expressed on immune cells which play a role in immune regulation. The aims of the present study were to determine whether circulating soluble T cell immunoglobulin and mucin domain-3 (sTIM-3) is elevated in rheumatoid arthritis (RA) patients, and investigate the relationships between STIM-3 and clinical features of RA.

\section{Methods}

The study included 116 patients with established RA and 27 healthy control subjects. Serum levels of sTIM-3 were measured via the enzyme-linked immunosorbent assays (ELISA). Correlations between serum STIM-3 and a range of parameters including ACPA titer, erythrocyte sedimentation rate (ESR) and matrix metalloproteinase-3 (MMP-3) were assessed.

\section{Results}

Serum STIM-3 was significantly elevated in RA patients compared with healthy subjects, and it was positively correlated with ACPA titer $(r=0.27 p=0.005)$, ESR $(r=0.27, p=0.004)$ and MMP-3 $(r=0.35, p<$ 0.001). In RA patients with high ACPA titers ( $\geq 200 \mathrm{U} / \mathrm{mL}$ ), sTIM-3 was not correlated with ESR or MMP-3. Whereas, sTIM-3 was significantly correlated with ESR and MMP-3 in RA patients with low ACPA titers (< $200 \mathrm{U} / \mathrm{mL})$.

\section{Conclusions}

Serum sTIM-3 was increased in RA patients, and it was associated with proinflammatory markers and disease activity in RA patients under a particular ACPA status. Our data suggest that circulating sTIM-3 may be a useful biomarker for the determination of disease activity in RA patients.

\section{Background}

Rheumatoid arthritis (RA) is a chronic systemic autoimmune disease characterized by synovitis and subsequent inflammatory bone destruction [1]. An accumulation of studies indicate that imbalance between innate and adaptive immune systems can lead to excessive immune responses in rheumatoid synovium [2]. Coinhibitory receptors such as cytotoxic T-lymphocyte-associated protein 4 have an important role in the regulation of T cell responses [3] and are evidently effective targets in RA, in that coinhibitory $T$ cell receptor expression can dampen effector $T$ cell responses [4]. 
The "next wave" of coinhibitory receptor targets are currently being explored in chronic diseases [5]. T cell immunoglobulin and mucin domain-3 (TIM-3) is a cell surface molecule that is expressed by $\mathrm{CD} 4^{+} \mathrm{T}$ helper (Th1) cells [6]. Functional studies investigating TIM-3 suggest that it is a negative regulator of Th1 immune responses [7]. In support of this, anti-TIM-3 antibody was shown to exacerbate experimental autoimmune diseases such as experimental autoimmune encephalomyelitis [8]. The C-type lectin galectin 9 was subsequently identified as a TIM-3 ligand [9]. Galectin-9-mediated ligation of TIM-3 has been shown to induce cell death in TIM-3-expressing Th1 cells, leading to the amelioration of autoimmune diseases [10]. TIM-3 was originally detected in adaptive immune cells, but following study demonstrated that TIM-3 is also expressed in innate immune cells [11]. Expression of TIM-3 was detected in osteoclasts and its mononuclear precursor cells in rheumatoid synovium, and the TIM-3/galectin-9 regulatory system controls osteoclastogenesis and inflammatory bone destruction in RA [12]. These findings suggest that rheumatoid inflammatory bone destruction may be influenced by upregulation of the TIM-3/galectin-9 axis. TIM-3 can be shed from the cell surface via a disintegrin and metalloproteinase with thrombospondin motifs (ADAMTS) 10-mediated or ADAM 17-mediated cleavage at the TIM-3 stalk region, resulting in a soluble form of TIM-3 (sTIM-3) that can be detected in serum [13]. We hypothesized that the TIM-3 axis is dysregulated in RA, and may be involved in rheumatoid inflammatory processes. In the current study serum levels of STIM-3 were investigated in RA patients, and associations between sTIM-3 and clinical RA parameters were analyzed.

\section{Methods}

\section{Patients}

This observational single-center study included 116 consecutive RA patients. Patients were enrolled between February 2012 and September 2019, with follow-up ending in September 2019. We retrospectively reviewed the records of these RA patients. All patients were treated in Department of Rheumatology, Fukushima Medical School from June 2009 to March 2019. All the patients met the 2010ACR/EULAR classification criteria for the disease [14]. Probable RA or overlap syndromes were excluded.

The following clinico-demographic data were collected from the Medical Records Unit at Fukushima University Hospital: age, age at onset of RA, gender, Disease Activity Score-28 for Rheumatoid Arthritis with erythrocyte sedimentation rate (ESR) (DAS28-ESR) score [15]. As controls, 27 healthy subjects (11 males, 16 females, median age 40 years, interquartile range [IQR]; 35-49 years) were included. This study was conducted in accordance with the principles of the Declaration of Helsinki. Ethical approval for this study (No. 2019097) was provided by the Ethics Committee of Fukushima Medical University.

\section{Measurement Of Clinical Disease Activity}


All patients underwent clinical assessment at baseline, including 28-joint swollen and tender joint counts (28-SJC and 28-TJC, respectively), physician and patient global assessment with visual analogue scales $(0-100 \mathrm{~mm})$ and ESR $(\mathrm{mm})$. The composite disease activity indices were subsequently calculated: DAS28-ESR [15]. Result of this score was reported in quantitative value divided into 4 categories: remission with score of $<2.6$, mild activity if score of $\geq 2.6$ to $<3.2$, moderate activity if score of $\geq 3.2$ to $<5.1$ and high activity if score of $\geq 5.1$. The patients' anti-CCP antibodies were analyzed using commercially available second-generation chemiluminescent enzyme immunoassay kits (STACIA ${ }^{8}$ MEBLux ${ }^{\text {TM }}$ CCP test, Medical and Biological Laboratories, Aichi, Japan) according to the manufacturer's instructions. Radiographs were taken of both hands of each patient. Two rheumatologists, blinded to the patient's identify and functional status, independently graded each hand radiographs and assigned as Steinbrocker radiographic stage [16].

\section{ELISA Methods}

Serum concentrations of sTIM-3were measured using enzyme-linked immnunosorbent assay kit (R\&D Systems, Minneapolis, MN, USA) according to the manufacturer's instruction.

\section{Statistical analysis}

Results were non-normally distributed and are presented throughout the manuscript with median and interquartile range [IQR], and were compared by the Mann-Whitney $U$ test. Correlations between continuous variables were analyzed by the Spearman's rank correlation test. All data entry and statistical analyses were performed using SPSS Statistics version 22.0 (IBM, Armonk, NY). In all the analyses, a 2tailed $p<0.05$ was considered statistically significant.

\section{Results}

\section{Demographic data of enrolled RA patient}

The study included 116 RA patients and 27 healthy controls. The demographics and clinical characteristics of the RA patients are shown in Table 1. Among 116 patients with RA, 83 (71.6\%) were female and their mean age was 63.5 years. The majority of the RA patients were taking diseasemodifying anti-rheumatic drugs (DMARDs), mostly methotrexate (59/116 50.9\%), and biologics (38/116 $32.8 \%)$.

\section{Serum sTIM-3 Concentrations In RA Patients}

Serum sTIM-3 levels were significantly higher in RA patients than they were in the healthy subjects (Fig. 1). There were significant positive correlations between serum sTIM-3 and rheumatoid inflammatory 
markers such as erythrocyte sedimentation rate $(E S R, r=0.27, p=0.004)$, matrix metalloproteinase-3 (MMP-3, $r=0.35, p<0.001)$, and ACPA titers $(r=0.27 p=0.005)$.

\section{Relationships Between sTIM-3 And ACPA Titers}

To further evaluate the capacity of serum STIM-3 to differentiate between RA phenotypes, distribution patterns of serum sTIM-3values were analyzed in conjunction with anti-citrullinated peptide antibody (ACPA) titers. In a two-dimensional map consisting of serum STIM-3 and ACPA titer, two distinct groups were apparent (Fig. 2). The cut-off values of ACPA titers $(200 \mathrm{U} / \mathrm{ml})$ was determined according to the ability to discriminate the differential correlations between STIM-3 and ACPA titer. There was a significant modest correlation between STIM-3 and ACPA titer in RA patients with high ACPA titers $(\geq 200 \mathrm{U} / \mathrm{mL} \mathrm{r}=$ $0.508, p=0.002)$. Whereas, there was no correlation between STIM-3 and ACPA titer in RA patients with low ACPA titers ( $<200 \mathrm{U} / \mathrm{mL}$ ). There was differential correlations between serum sTIM-3 levels and ACPA titers in these two groups, suggesting that STIM-3 could be modulated by ACPA titers.

Relationships between sTIM-3 and clinical and laboratory parameters (Fig. 3)

In RA patients with low ACPA titers $(<200 \mathrm{U} / \mathrm{mL})$, serum sTIM-3 was significantly correlated with the inflammatory markers, ESR $(r=0.36, p=0.001)$ and MMP-3 $(r=0.38, p<0.001)$. Whereas corresponding correlations were not significant in RA patients with high ACPA titers ( $\geq 200 \mathrm{U} / \mathrm{mL}$ ). These findings suggest that serum STIM-3 upregulation may be associated with autoimmune responses in RA patients with high ACPA titers, whereas serum STIM-3 was upregulated in response to inflammatory mediators in RA patients with low ACPA titers.

In RA patients with low ACPA titers ( $<200 \mathrm{U} / \mathrm{mL}$ ), circulating sTIM-3 was significantly higher in CRPpositive patients than it was in CRP-negative patients (2871 pg/mL, [IQR 2145-3921] versus. $2081 \mathrm{pg} / \mathrm{mL}$, [IQR 1714-2840], $p=0.007)$. Conversely, in patients with high ACPA titers $(\geq 200 \mathrm{U} / \mathrm{mL})$ there was no significant difference in circulating sTIM-3 between CRPpositive and CRP-negative patients (2998 pg/mL, [IQR 2640-3960] versus. $2385 \mathrm{pg} / \mathrm{mL}$, [IQR 1712-3826], $p=0.20$ ).

\section{Relationships Between sTIM-3 And RA Clinical Outcomes}

From a clinical point of view, the circulating STIM-3 were compared according to the presence or absence of clinical remission in RA patients (Fig. 4A). Serum sTIM-3 was significantly higher in RA patients without clinical remission compared to those with clinical remission (2852 pg/ml [IQR: 2013-3584] versus $1954 \mathrm{pg} / \mathrm{ml}$ [IQR: 1611-2875], $p=0.005)$. As shown in Fig. 4B, RA patients with advanced joint damage (stage III or IV) had significantly higher levels of serum STIM-3 than those without advanced joint damage (2830 pg/ml [IQR: 2181-4047] versus $2428 \mathrm{pg} / \mathrm{ml}$ [IQR: 1927-3175], $p=0.011)$. There was a differential relationship between circulating STIM-3 and rheumatoid inflammatory markers in the presence or absence of advanced joint damage (stage III or IV). In RA patients with advanced joint 
damage (stage III or IV), serum sTIM-3 significantly correlated with MMP-3 but not with ESR. Conversely, in RA patients without advanced joint damage, circulating STIM-3 was significantly correlated with ESR, whereas there was a weak correlation between sTIM-3 and MMP-3 (Fig. 5).

\section{Discussion}

RA is the most common autoimmune disease characterized by immune-mediated etiology associated with synovial inflammation and articular destruction [17]. Genetic and environmental factors play a role in RA risk, and complex interactions between multiple cytokines, immune cells, and biological pathways contribute to RA development [18]. Intricate relationships between genetic, environmental, and cellular factors may contribute to the heterogeneity of RA [19]. Few clinically useful biomarkers exist for RA in terms of disease phenotype, treatment response, and disease progression. ACPA production tends to be associated with a more aggressive course [20], but this is by no means inevitable and other prognostic markers are also involved in RA phenotypes [21]. New biomarkers for RA disease phenotypes, joint outcomes, and severity may improve therapeutic outcomes in RA patients. Various RA-related biomarkers have been identified, but which of these are the most informative with regard to immunomodulatory factors and RA phenotypes remains to be established [22].

A growing body of evidence pertaining to contributions of co-inhibitory receptor pathways to immunemediated disorders has expanded to include TIM-3 [23]. Our proposed hypothesis is that the TIM$3 /$ galectin-9 axis is implicated in maintaining the regulation of adaptive and innate immune responses in RA. In the current study we investigated serum levels of sTIM-3 in patients with established RA. Circulating STIM-3 was significantly elevated in RA patients and it was correlated with ACPA titers and rheumatoid inflammatory markers, reflecting the activation of adaptive and innate immunity. In RA patients with low ACPA titers, there were positive correlations between STIM-3 and rheumatoid inflammatory markers. Whereas neither of these parameters was significantly associated with sTIM-3 in RA patients with high ACPA titers. These findings suggest that circulating STIM-3 levels are differentially regulated in RA patients depending on their ACPA status.

TIM-3 is a surface molecule expressed on immune cells that plays an important role in immune regulation [9]. Identification of galectin-9 as a TIM-3 ligand has established the TIM-3/galectin-9 pathway as a regulator of Th1 immunity [7]. Ligation of TIM-3 by galectin9 in a mouse model of collagen-induced arthritis effectively inhibited the severity of the condition, including inflammatory cell infiltration and bone distinction [10]. Conversely, blocking the TIM-3/galectin-9 pathway via a monoclonal antibody or gene knockout can reportedly exacerbate autoimmune conditions [24]. High expression of TIM-3 in synovial tissues and peripheral mononuclear cells has been demonstrated in RA patients [25]. More recently, we and other group demonstrated the elevated serum levels of Galectin-9 in RA patients[26, 27]. Furthermore, beneficial effect of galectin-9 on rheumatoid arthritis through the induction of apoptosis of synovial fibroblasts had been suggested [28]. It can be concluded that the TIM-3/galectin-9 pathway is activated in RA as an anti-immune mediators. However, this pathway can be modulated by the soluble form of TIM-3 which is shedded form TIM-3 expressing immune cells[13, 29]. TIM-3/galectin-9 interaction may result in 
T cell exhaustion, on the contrary, sTIM-3 seems to have alternative effects against this feedback mechanism. Given the apparent complexity of the function of TIM-3, further studies are needed to determine the source of serum STIM-3 and its role in rheumatoid inflammatory progression in RA patients.

Predicting drug responses and clinical courses in RA patients is challenging due to the heterogeneity of individual clinical phenotypes. ACPA-positive and ACPA-negative patients may exhibit similar clinical manifestations at baseline, but different phenotypes with respect to subsequent radiographic progression [30]. Interestingly, in the present study there was a modest correlation between sTIM-3 concentration and MMP-3 in RA patients with advance joint damage (stage III or IV). RA is characterized by two key events, immune activation and subsequent inflammatory bone damage [31]. MMP-3 is mainly produced by rheumatoid synovial fibroblasts or osteoclasts activated by inflammatory cytokines, and it can degrade components of the cartilage extracellular matrix (ECM), leading to rheumatoid bone destruction [32]. Therefore, the modest correlation between STIM-3 and MMP-3 in RA patients with advanced bone damage suggest that STIM-3 upregulation may reflect the activated status of rheumatoid stromal cells, including MMP-3 induction, under the rheumatoid bone destruction processes.

There are several potential limitations of this study that should be considered. First, the patient population was relatively small and a larger study is essential to confirm our results. Second, all patients with RA and healthy individuals in this study were Japanese, additional studies in other ethnic groups are needed to verify these findings. Third, it will be important to examine the longitudinal changes of serum sTIM-3 levels in patients with RA and to assess their clinical course in the future studies

\section{Conclusions}

Circulating sTIM-3 was significantly higher in RA patients without clinical remission compared with those with clinical remission as well as healthy subjects. Circulating STIM-3 significantly correlated with rheumatoid inflammatory markers or matrixdegrading markers in RA patients under particular ACPA status, or degrees of joint damage. Combined consideration of sTIM-3 and ACPA titers may facilitate distinctions between degrees of RA disease activity.

\section{Abbreviations}

ADAMTS: a disintegrin and metalloproteinase with a thrombospondin type 1 motif; ACPA: anticitrullinated peptide antibody; DAS28: disease activity score 28; IGR: interquartile range; MMP-3: matrix metalloproteinase-3; RA:rheumatoid arthritis; STIM-3: soluble T cell immunoglobulin domain and mucin-3; Th1:T helper 1

\section{Declarations}

\section{Ethical Approval and Consent to participate}


Ethical approval for this study (No. 2019097) was provided by the Ethics Committee of Fukushima Medical University.

\section{Consent for publication}

Not applicable

\section{Availability of supporting data}

Data sharing is not applicable to this article as no datasets were generated or analysed during the current study.

\section{Competing interests}

$\mathrm{KM}$ has received research grants from Chugai, Pfizer, and AbbVie. Rest of the authors declares that they have no competing interests

\section{Funding}

The study was supported by the Japan Grant-in-Aid for Scientific Research(C 17K09981)

\section{Authors' contributions}

HM, YF, TA, NM, JT, SS, MF, HW were involved in acquisition of clinical data. YF and KM drafted manuscript.

YF, KM carried out the biochemical studies, participated in the sequence alignment and drafted the manuscript. HM, KM participated in the sequence alignment and drafted the manuscript. HM, KM participated in the design of the study, FY performed the statistical analysis. All authors read and approved the final manuscript.

\section{Acknowledgements}

We are grateful to Ms Kanno Sayaka for her technical assistance in this study.

\section{References}

1. Townsend MJ. Molecular and cellular heterogeneity in the Rheumatoid Arthritis synovium: clinical correlates of synovitis. Best Pract Res Clin Rheumatol. 2014;28(4):539-49.

2. Catrina Al, Joshua V, Klareskog L, Malmström V. Mechanisms involved in triggering rheumatoid arthritis. Immunol Rev. 2016;269(1):162-74.

3. Zhang Q, Vignali DA. Co-stimulatory and Co-inhibitory Pathways in Autoimmunity. Immunity. 2016;44(5):1034-51. 
4. Fiocco U, Sfriso P, Oliviero F, Pagnin E, Scagliori E, Campana C, et al. Co-stimulatory modulation in rheumatoid arthritis: the role of (CTLA4-Ig) abatacept. Autoimmun Rev. 2008;8(1):76-82.

5. Anderson AC, Joller N, Kuchroo VK. Lag-3. Tim-3, and TIGIT: Co-inhibitory Receptors with Specialized Functions in Immune Regulation. Immunity. 2016;44(5):989-1004.

6. Das M, Zhu C, Kuchroo VK. Tim-3 and its role in regulating anti-tumor immunity. Immunol Rev. 2017;276(1):97-111.

7. Anderson AC, Anderson DE. TIM-3 in autoimmunity. Curr Opin Immunol. 2006;18(6):665-9.

8. Monney L, Sabatos CA, Gaglia JL, Ryu A, Waldner H, Chernova T, et al. Th1-specific cell surface protein Tim-3 regulates macrophage activation and severity of an autoimmune disease. Nature. 2002;415(6871):536-41.

9. Zhu C, Anderson AC, Kuchroo VK. TIM-3 and its regulatory role in immune responses. Curr Top Microbiol Immunol. 2011;350:1-15.

10. Seki M, Oomizu S, Sakata KM, Sakata A, Arikawa T, Watanabe K, et al. Galectin-9 suppresses the generation of Th17, promotes the induction of regulatory $\mathrm{T}$ cells, and regulates experimental autoimmune arthritis. Clin Immunol. 2008;127(1):78-88.

11. Anderson AC, Anderson DE, Bregoli L, Hastings WD, Kassam N, et al. Promotion of Tissue Inflammation by the Immune Receptor Tim-3 Expressed on Innate Immune Cells. Science. 2007;318:1141-3.

12. Moriyama K, Kukita A, Li YJ, Uehara N, Zhang JQ, Takahashi I, et al. Regulation of osteoclastogenesis through Tim-3: possible involvement of the Tim-3/galectin-9 system in the modulation of inflammatory bone destruction. Lab Invest. 2014;94(11):1200-11.

13. Möller-Hackbarth K, Dewitz C, Schweigert O, Trad A, Garbers C, Rose-John S, et al. A disintegrin and metalloprotease (ADAM) 10 and ADAM17 are major sheddases of T cell immunoglobulin and mucin domain 3 (Tim-3). J Biol Chem. 2013;288:34529-44.

14. van der Linden MP, Knevel R, Huizinga TW, van der Helm-van Mil AH. Classification of rheumatoid arthritis: comparison of the 1987 American College of Rheumatology criteria and the 2010 American College of Rheumatology/European League Against Rheumatism criteria. Arthritis Rheum. 2011;63(1):37-42.

15. Fransen J, van Riel PL. The Disease Activity Score and the EULAR response criteria. Rheum Dis Clin North Am. 2009;35(4):745-57. vii-viii.

16. Steinbrocker $\mathrm{O}$, Traeger $\mathrm{CH}$, Batterman RC. Therapeutic criteria in rheumatoid arthritis. J Am Med Assoc. 1949;140:659-62.

17. Scott DL, Wolfe F, Huizinga TW. Rheumatoid arthritis. Lancet. 2010;376:1094-108.

18. Harre U, Schett G. Cellular and molecular pathways of structural damage in rheumatoid arthritis. Semin Immunopathol. 2017;39(4):355-63.

19. Sparks JA, Costenbader KH. Genetics, environment, and gene-environment interactions in the development of systemic rheumatic diseases. Rheum Dis Clin North Am. 2014;40(4):637-57. 
20. Willemze A, Trouw LA, Toes RE, Huizinga TW. The influence of ACPA status and characteristics on the course of RA. Nat Rev Rheumatol. 2012;8(3):144-52.

21. Szodoray P, Szabó Z, Kapitány A, Gyetvai A, Lakos G, Szántó S, et al. Anti-citrullinated protein/peptide autoantibodies in association with genetic and environmental factors as indicators of disease outcome in rheumatoid arthritis. Autoimmun Rev. 2010;9(3):140-3.

22. Trouw LA, Mahler M. Closing the serological gap: promising novel biomarkers for the early diagnosis of rheumatoid arthritis. Autoimmun Rev. 2012;12(2):318-22.

23. Sakuishi K, Jayaraman P, Behar SM, Anderson AC, Kuchroo VK. Emerging Tim-3 functions in antimicrobial and tumor immunity. Trends Immunol. 2011;32(8):345-9.

24. Takuma Isshiki H, Akiba M, Nakayama N, Harada K, Okumura S, Homma. Sachiko Miyake Anti-TIM-3 Treatment Exacerbates Pulmonary Inflammation and Fibrosis in Mice. J Immunol. 2017;199:3733-7.

25. Liu Y, Shu Q, Gao L, Hou N, Zhao D, Liu X, et al. Increased Tim-3 expression on peripheral lymphocytes from patients with rheumatoid arthritis negatively correlates with disease activity. Clin Immunol. 2010;137(2):288-95.

26. Wang Y, Song L, Sun J, Sui Y, Li D, Li G, Liu J, Shu Q.Wang Y, et al. Expression of Galectin-9 and correlation with disease activity and vascular endothelial growth factor in rheumatoid arthritis. Clin Exp Rheumatol. 2019 in press. PMID: 31820713.

27. Fujita Y, Asano T, Matsuoka N, Temmoku J, Sato S, Matsumoto H, et al. Differential regulation and correlation between galectin- 9 and anti-CCP antibody (ACPA) in rheumatoid arthritis patients. Arthritis Res Ther. 2020;22(1):80. doi:10.1186/s13075-020-02158-3.

28. Seki M, Sakata KM, Oomizu S, Arikawa T, Sakata A, Ueno M, et al. Beneficial effect of galectin 9 on rheumatoid arthritis by induction of apoptosis of synovial fibroblasts. Arthritis Rheum. 2007;56:3968-76.

29. Jones RB, Ndhlovu LC, Barbour JD, Sheth PM, Jha AR, Long BR, et al. Tim-3 expression defines a novel population of dysfunctional T cells with highly elevated frequencies in progressive HIV-1 infection. J Exp Med. 2008;205:2763-79.

30. Ferris RL, Lu B, Kane LP. Too much of a good thing? Tim-3 and TCR signaling in T cell exhaustion. J Immunol. 2014;193(4):1525-30.

31. Alivernini S, Galeazzi M, Peleg H, Tolusso B, Gremese E, Ferraccioli G, et al. Is ACPA positivity the main driver for rheumatoid arthritis treatment? Pros and cons. Autoimmun Rev. 2017;16(11):1096102.

32. Komatsu N, Takayanagiã̃H H. Immune-bone interplay in the structural damage in rheumatoid arthritis. Clin Exp Immunol. 2018;194(1):1-8.

33. Redlich K, Hayer S, Ricci R, David JP, Tohidast-Akrad M, Kollias G, et al. Osteoclasts are essential for TNF-alpha-mediated joint destruction. J Clin Invest. 2002;110(10):1419-27.

\section{Table}




\section{Figures}

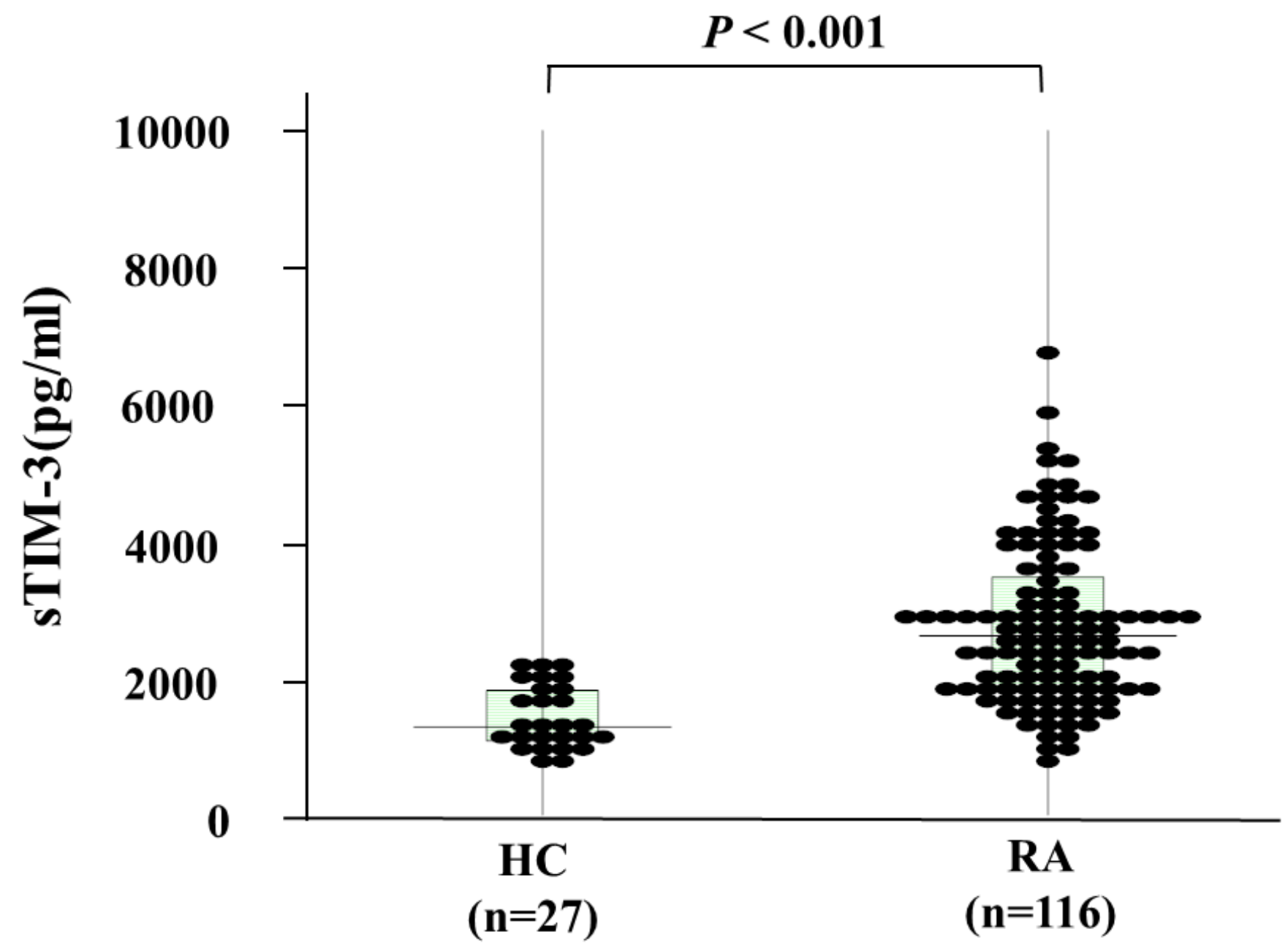

Figure 1

Serum levels of sTIM 3 in RA patients and controls. Serum levels of Galectin-9 in RA patients $(n=116)$ were significantly higher compared to those in healthy subjects $(n=27)$. 


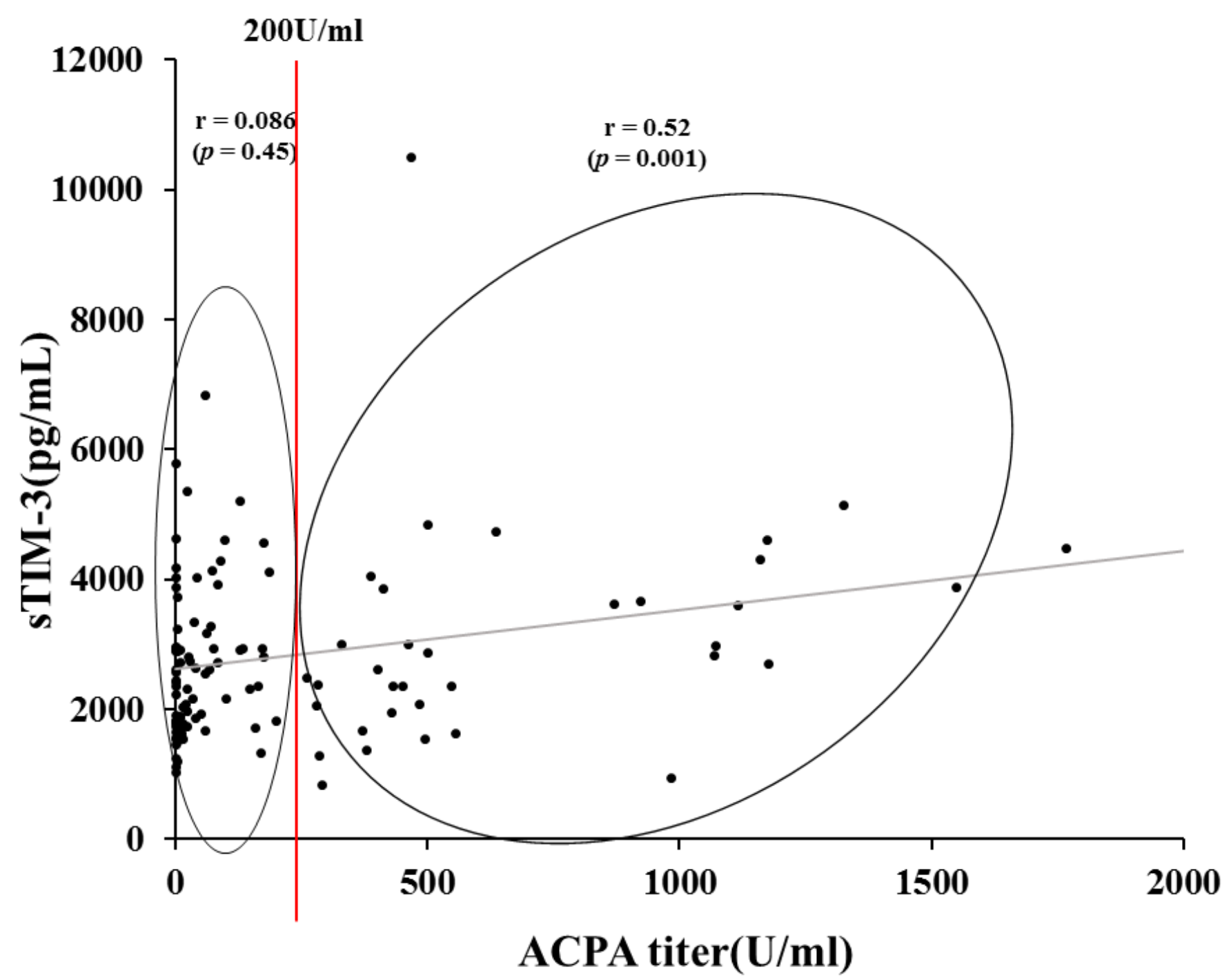

Figure 2

Relationship between anti-cirtrullinated peptide antibody (ACPA) titers and serum levels of sTIM 3 in patients with rheumatoid arthritis (RA). Levels of ACPA titers were measured and correlation analysis with serum levels of STIM 3 was performed. There was no significant correlation between serum levels of

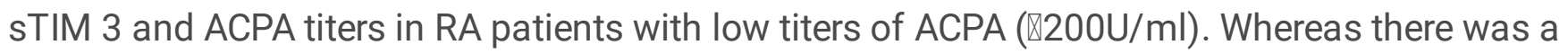
significant modest correlation between serum levels of STIM 3 and ACPA titers in RA patients with high titers of ACPA ( $\geqq 200 \mathrm{U} / \mathrm{ml})$. 

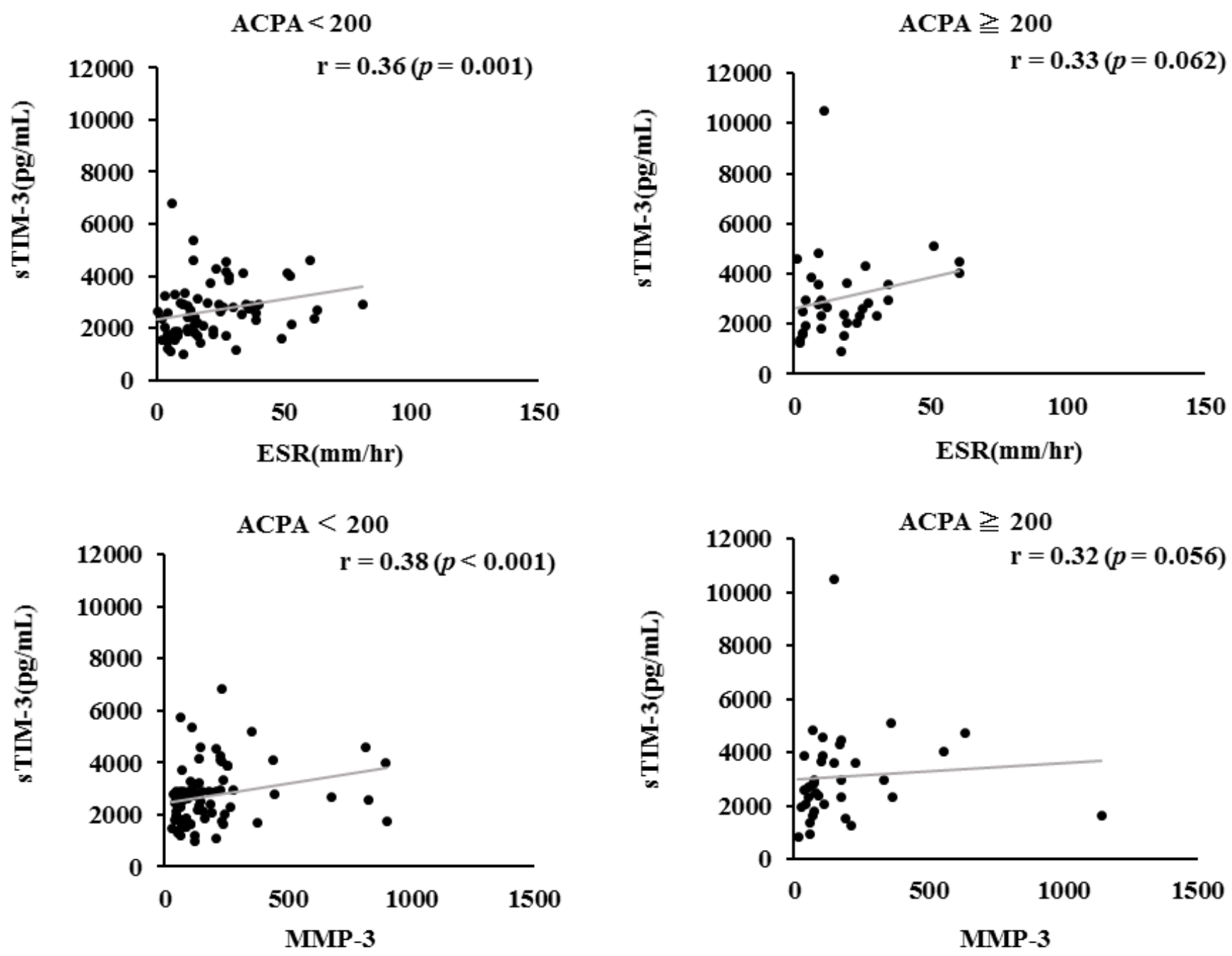

Figure 3

Correlation between serum levels of sTIM 3 and proinflammatory markers (A: ESR, B: MMP-3) in the subgrouped RA patients according to the titers of ACPA. Correlation analysis of serum levels of STIM 3 and clinical parameters (A: ESR, B: MMP-3) does not show a relationship in RA patients with high titers of ACPA ( $\geqq 200 U / m l)$. Whereas there was a significant positive correlation between clinical parameters (A: ESR, B: MMP-3) in RA patients with low titers of ACPA ( $8200 \mathrm{U} / \mathrm{ml})$. 

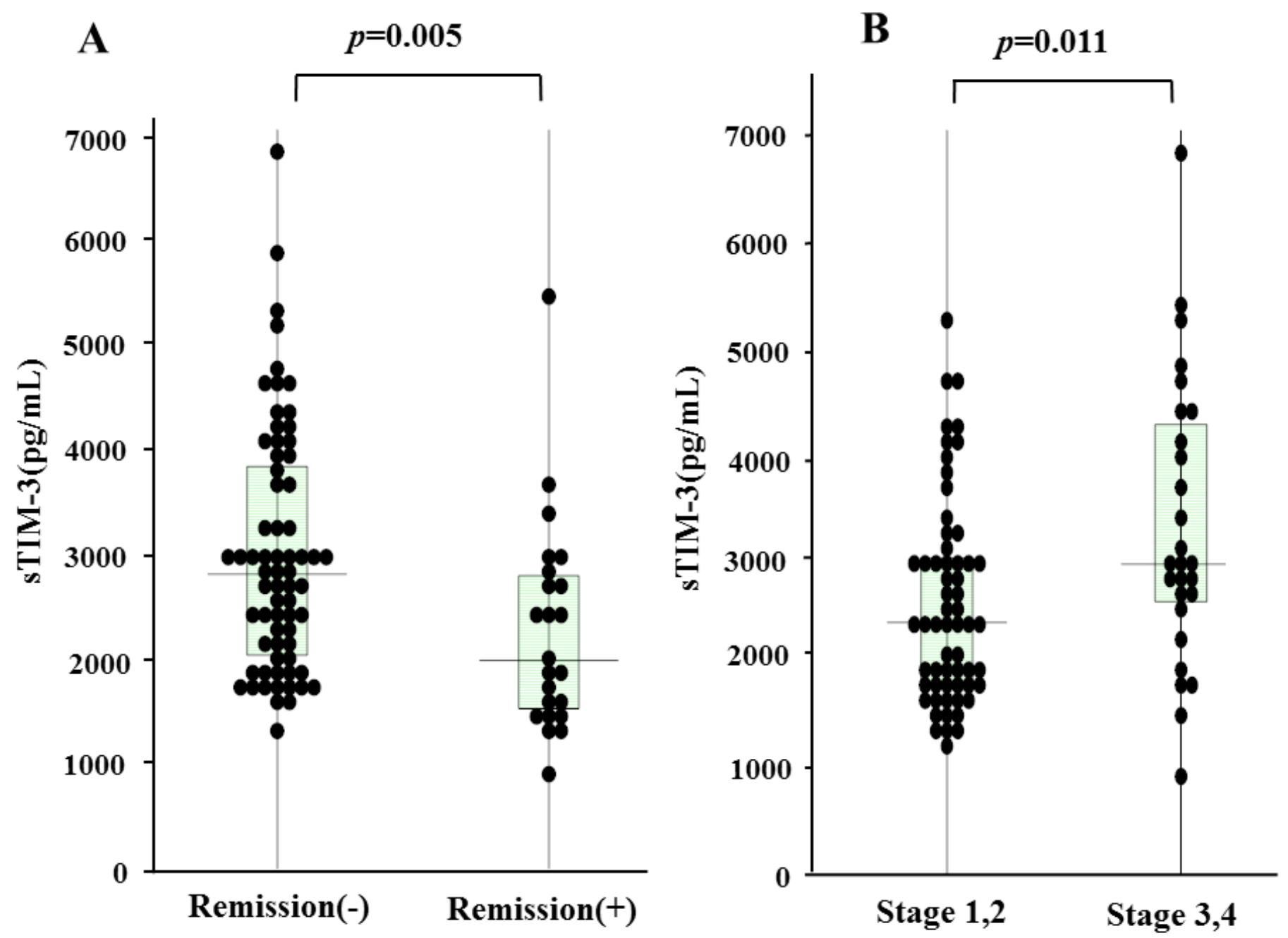

Figure 4

A Serum levels of sTIM 3 were compared between RA patients with or without DAS28-ESR clinical remission (A) or those with or without advanced joint damage (B). A; Serum STIM 3 were significantly lower in RA patients with $\mathrm{CR}$ compared to those without $\mathrm{CR}$. $\mathrm{CR}=$ clinical remission B: Serum STIM 3 were significantly higher in RA patients with advance joint damage (stage III or IV) compared to those without advance joint damage 

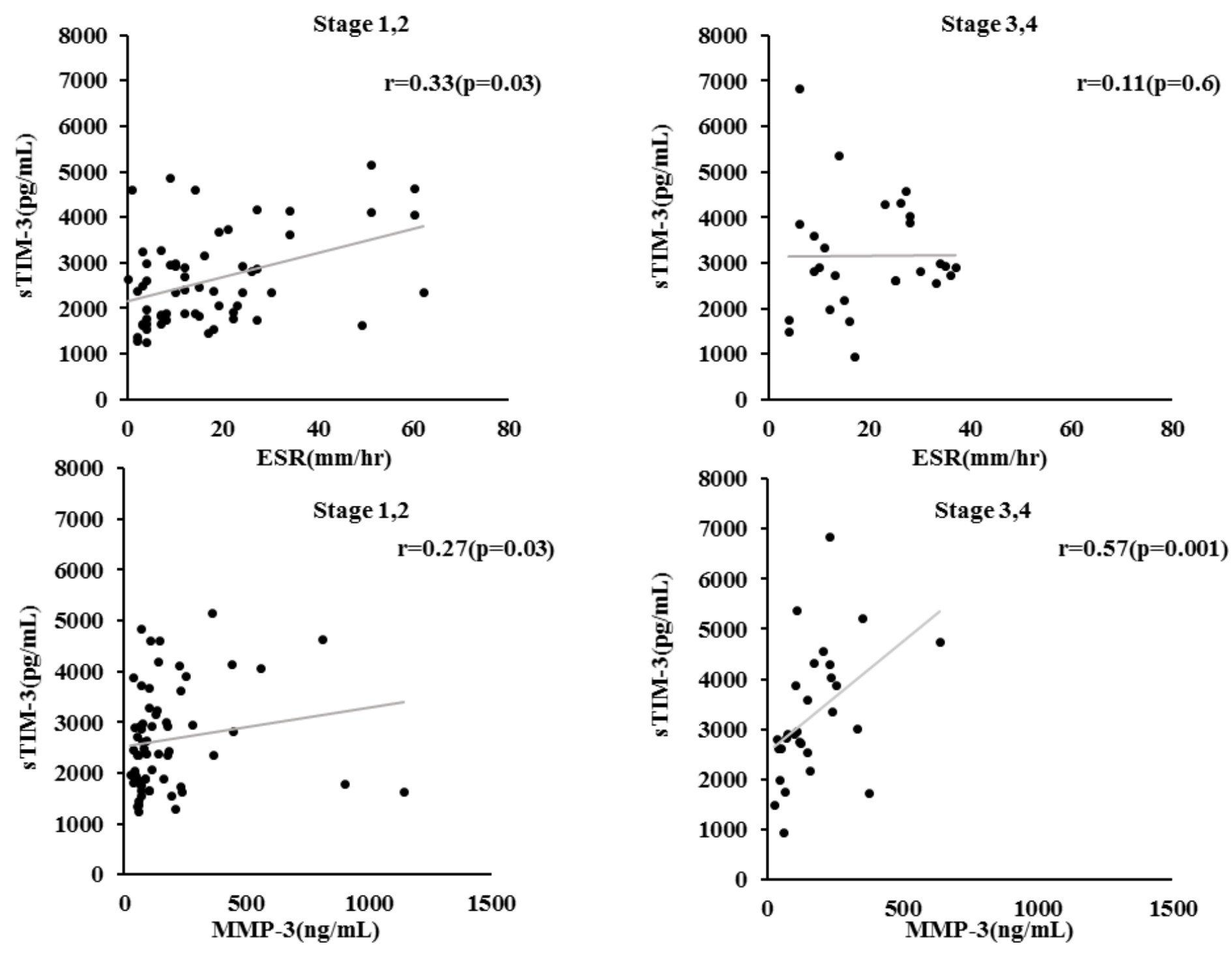

Figure 5

Correlation between serum levels of sTIM 3 and proinflammatory markers (A: ESR, B: MMP-3) in the subgrouped RA patients according to the advanced joint damage. Serum STIM 3 significantly correlated with ESR not with MMP-3 in RA patients without the advanced joint damage (stage III or IV). Whereas there was a significant positive correlation between STIM 3 and MMP-3, not with ESR in RA patients with the advanced joint damage.

\section{Supplementary Files}

This is a list of supplementary files associated with this preprint. Click to download.

- renamedc0ac6.pdf 